\title{
High-energy shift of the Urbach ultraviolet absorption from attenuated dynamical disorder in fluorine modified sol-gel silica
}

\author{
A. Paleari, ${ }^{a}$ F. Meinardi, A. Lauria, R. Lorenzi, and N. Chiodini \\ Department of Materials Science, University of Milano-Bicocca, Consorzio Nazionale Interuniversitario per \\ le Scienze Fisiche della Materia (CNISM), Via R. Cozzi 53, I-20125 Milano, Italy \\ S. Brovelli \\ Department of Physics and Astronomy, London Centre for Nanotechnology, University College London, \\ London WC1E 6BT, United Kingdom
}

(Received 12 June 2007; accepted 15 September 2007; published online 3 October 2007)

\begin{abstract}
Fluorine modified amorphous silica has been synthetized via sol-gel route and studied through analysis of the temperature dependence of the Urbach absorption tail in the vacuum-ultraviolet region. The modified glass has a steep absorption edge above $8 \mathrm{eV}$, with the absorption coefficient $\alpha \propto \exp \left[E / E_{U}(T)\right]$ showing Urbach energy values $E_{U}(T)$ ranging between 50 and $66 \mathrm{meV}$. The comparison of $E_{U}(T)$ with pure silica data indicates a structural softening caused by the reduction of dynamical disorder, and suggests that the F-modified sol-gel synthesis is an appropriate route for achieving high energy shifts of the absorption edge. (C) 2007 American Institute of Physics.
\end{abstract}

[DOI: 10.1063/1.2794429]

Several technological areas are looking for new material compositions and synthetic routes for the production of optical materials with transmission window extending into the vacuum ultraviolet (vuv) region and with improved radiation hardness and thermal stability. ${ }^{1,2}$ In specific applications such as photomasks for lithography at $157 \mathrm{~nm}$ and materials for vuv sources, silicalike structural features are unique in assuring low thermal expansion coefficient and good workability. ${ }^{3}$ For these reasons, modifications of silica optical properties through specific synthesis or doping processes are currently being investigated to extend its operational window into the vuv. Fluorine modified silica-based materials are promising candidates for these applications, since the silica absorption edge shifts toward high energy after fluorine doping. ${ }^{4-8}$ The structural origin of this effect was analyzed in F-modified glasses from chemical vapor deposition (CVD) methods, identifying different roles of fluorine, mainly in the modification of the mean bond energy and the structural disorder of the amorphous matrix. ${ }^{5,9-11}$ In this scenario, fabrication of F-modified silica from sol-gel synthesis ${ }^{12}$ should, in principle, be an interesting target because of the relatively lowcost and sustainable features of the process, and for the expected improvement in radiation hardness with respect to other types of silica. ${ }^{13,14}$ However, only few studies on F-doped sol-gel silica have been reported regarding preforms for low-index optical fibers, ${ }^{15}$ and no vuv data have been published on fluorinated silica from sol-gel synthesis. In fact, sol-gel silica usually contains a large number of $\mathrm{OH}$ groups that are responsible for a broad absorption band at $8 \mathrm{eV},{ }^{16}$ which is strongly detrimental for vuv applications. In the present work, we disclose a method for the fabrication of optical grade bulk samples of F-doped dry silica by means of a modified sol-gel method. Our results show that the achievable reduction of dynamical disorder and $-\mathrm{OH}$ content opens the way to the utilization of sol-gel materials in vuv applications.

\footnotetext{
${ }^{\text {a)} E l e c t r o n i c ~ m a i l: ~ a l b e r t o . p a l e a r i @ m a t e r . u n i m i b . i t ~}$
}

Bulk samples, about $0.5 \mathrm{~mm}$ thick, of fluorine-modified silica were prepared by the sol-gel technique, cogelling tetraethoxysilane and trimethoxyfluorosilane, with concentration ratio 1:9. After gelation and drying, the xerogel sample was heated in oxygen (ramping at $3^{\circ} \mathrm{C} / \mathrm{h}$, up to $1100{ }^{\circ} \mathrm{C}$ ) to induce both fluorine activated drying and fluorine perturbed densification of the silica network. Optical and structural features of the final glass have been analyzed by means of refractive index measurements at $633 \mathrm{~nm}$ with $10^{-4}$ resolution, Raman scattering and infrared absorption spectroscopy, with a resolution of $0.1 \mathrm{~cm}^{-1}$. vuv transmission data have been collected by means of synchrotron radiation at the SUPERLUMI experimental station of HASYLAB (DESY, Hamburg), with $0.2 \mathrm{~nm}$ spectral resolution. The spectral distribution of the intensity $I_{c}$, collected behind the sample along the incident beam direction has been compared with the incident intensity $I_{0}$, measured in the same configuration without the sample. The contribution $I_{s}$ from scattered light has been quantified from the background signal at negligible sample transmittance. The absorption coefficient $\alpha$ has been obtained from $\alpha=(1 / L) \ln \left[\left(I_{0}-I_{s}\right)(1-R)^{2} /\left(I_{c}-I_{s}\right)\right]$, where $L$ is the sample thickness and $(1-R)^{2}$ is the correction factor for the reflectivity $R$ at normal incidence. $R$ has been evaluated at $633 \mathrm{~nm}$ from the refractive index $n$, whereas the spectral dependence $R(\lambda)$ [inset of Fig. 1(a)] has been derived from $n(\lambda)$ calculated in F-modified silica ${ }^{4}$ with congruent $n$ value at $633 \mathrm{~nm}$ [see Fig. 1(a) where a few sets of data are compared and fitted with the Sellmeier relation]. ${ }^{4,17}$ In the Urbach region $(8-8.4 \mathrm{eV})$, errors on $\alpha$ from the uncertainty in $(1-R)^{2}$ are smaller than $2 \%$.

The Raman spectrum in Fig. 1(b) shows that the synthesis gives rise to a fully densified silicalike network. Spectral features at 440, 490, 606, 800, 1050 , and $1160 \mathrm{~cm}^{-1}$ correspond to silica Raman modes $\omega_{1}, D_{1}, D_{2}, \omega_{3}, \omega_{4}(\mathrm{LO})$, and $\omega_{4}(\mathrm{TO})$, respectively. ${ }^{18}$ The peak at $945 \mathrm{~cm}^{-1}$, ascribable to $\mathrm{Si}-\mathrm{F}$ stretching mode, ${ }^{11}$ is an evidence of fluorine in $\equiv \mathrm{Si}-\mathrm{F}$ sites. The relative intensity is consistent with a $\mathrm{F}$ concentration of the order of $10^{4} \mathrm{ppm},{ }^{11}$ in agreement with the value estimated from the refractive index compared with data from 


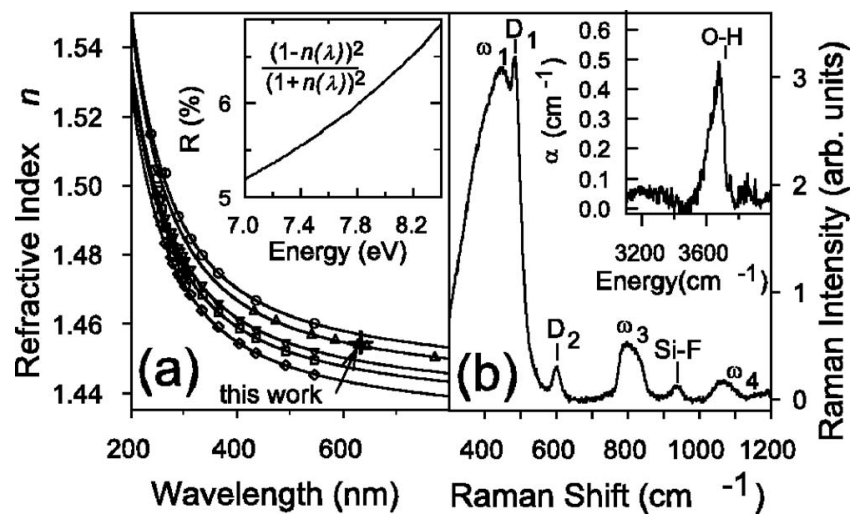

FIG. 1. (a) Refractive index $n$ at $633 \mathrm{~nm}$ in F-modified sol-gel silica (cross mark) compared with data by Tsukuma et al. (Ref. 17) on $0 \%(\bigcirc), 2.1 \%$ $(\nabla), 2.6 \%(\square)$, and $3.7 \%(\diamond)$ F-doped silica, and by Smith and Moore (Ref. 4) on $0.8 \%$ F-doped silica $(\triangle)$; lines are calculated dispersion curves. Inset: normal incidence reflectivity in the vuv range calculated from $n(\lambda)$ of $0.8 \%$ F-doped silica. (b) Raman spectrum of F-modified sol-gel silica. Inset: infrared absorption in the range of $\mathrm{O}-\mathrm{H}$ stretching mode.

the literature in Fig. 1(a). ${ }^{4,17}$ This amount is about a factor 10 lower than the initial concentration of fluorinated Si molecular precursors in the sol-gel synthesis. A large fraction of fluorinated precursors are indeed released during the thermal treatment through reaction with hydroxyl groups embedded in the sol-gel network. ${ }^{19}$ As a result, the fluorinated network is strongly dried with respect to typical sol-gel silica where the content of $-\mathrm{OH}$ groups is a few thousand ppm. ${ }^{12}$ From the infrared spectrum in the inset of Fig. 1(b), the remaining $-\mathrm{OH}$ content is estimated ${ }^{20}$ to be $16 \pm 2 \mathrm{ppm}$, two orders of magnitude lower than in typical sol-gel silica. vuv absorption spectra in Fig. 2 indeed show a contribution at $8 \mathrm{eV}$, superimposed on the absorption edge, ascribed to electronic transitions of silanol groups, ${ }^{1,8,16}$ and whose cross section is $5 \times 10^{-19} \mathrm{~cm}^{2}$. The $8 \mathrm{eV}$ absorption in Fig. 2 indicates a silanol content of $10-20 \mathrm{ppm}$, in agreement with the infrared analysis.

Looking at the Urbach tail in Fig. 2, three facts may be remarked: (i) the edge is steeper than in synthetic silica (dashed curve reported from the literature ${ }^{5}$ ); (ii) contributions from point defects (specifically at $7.6 \mathrm{eV}$ ) ${ }^{8,21,22}$ are not detected; and (iii) the optical gap widens much more with

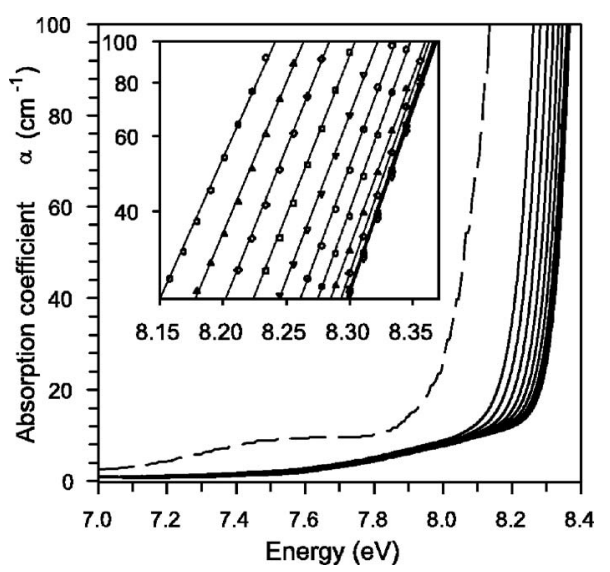

FIG. 2. vuv absorption spectra of F-modified sol-gel silica at temperatures from 300 to $25 \mathrm{~K}$, in step of $25 \mathrm{~K}$ from left to right, compared with dry pure silica at $300 \mathrm{~K}$ [dashed line, from Skuja et al. (Ref. 8)]. Inset: exponential region of the Urbach tail with absorption coefficient on a log scale. Straight lines are linear regression of the data.

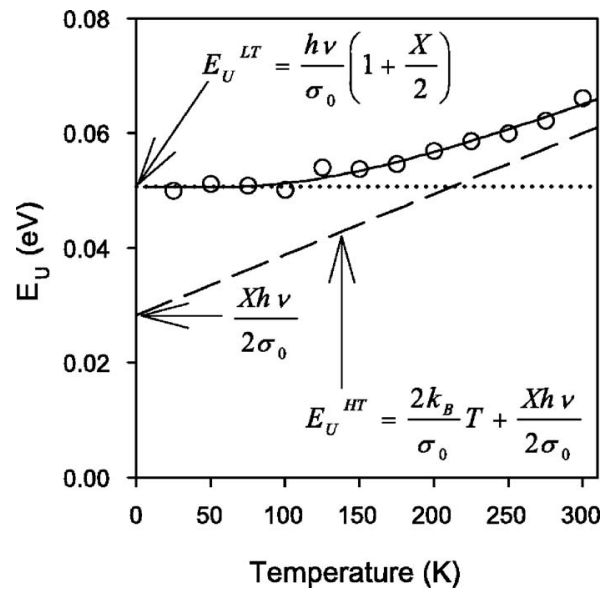

FIG. 3. Temperature dependence of the Urbach energy $E_{U}$ (circles) calculated from the data in Fig. 2. Fitting curves within the Einstein approximation (full line), with high-temperature $E_{U}^{\mathrm{HT}}$ and low-temperature $E_{U}^{\mathrm{LT}}$ regimes (dashed and dotted lines, respectively), are indicated.

decreasing temperature than in pure silica, ${ }^{22}$ down to temperatures less than $100 \mathrm{~K}$. These features may be analyzed in detail by looking at the Urbach behavior ${ }^{8,22}$ of the absorption coefficient $\alpha(E, T) \propto \exp \left[E / E_{U}(T)\right]$ as a function of the energy $E$, where $E_{U}$ accounts for the broadness of the energy band tail caused by static and dynamical disorder on the electronic density of states. ${ }^{22,23}$ So, the lower $E_{U}$, the sharper and steeper the edge, with a broader spectral window of transmission. In the inset of Fig. 2, we report the absorption coefficient on a semilog scale in the restricted spectral range of the Urbach tail, together with linear fits of the data. The curves shift toward higher energy with decreasing temperature, and this shift is accompanied by an increase in their slope. Indeed, within the experimental error, the curves converge at a common point at $8.75 \mathrm{eV}$, as expected with the Urbach approach. ${ }^{23}$ Figure 3 shows the calculated $E_{U}(T)$ at different temperatures. The values are significantly lower than in pure silica ${ }^{22}$ (50 meV instead of $73 \mathrm{meV}$ at low temperature and $66 \mathrm{meV}$ compared with $75 \mathrm{meV}$ at $300 \mathrm{~K}$ ), with a steeper increase with the temperature. Indeed, $E_{U}(T)$ reaches the low temperature quantum limit only below $100 \mathrm{~K}$ instead of $200 \mathrm{~K}$ in pure silica. ${ }^{22}$ The data follow the function $E_{U}=[h \nu \operatorname{coth}(h \nu / 2 k T)+X h \nu / 2] / \sigma_{0}($ Ref. 22) (with $h \nu=37 \mathrm{meV}, X=2.5$, and $\sigma_{0}=1.6$ ), where static and dynamic contributions to the Urbach energy are explicitly accounted for. The term with $X$ is the temperature independent contribution from static structural disorder, whereas the term with $\operatorname{coth}(h \nu / 2 k T)$ describes, within the Einstein approximation, ${ }^{23}$ the thermal broadening of the tail caused by electron-phonon coupling. The energy $h \nu$ is the characteristic value of the Einstein approximation, corresponding to an average phonon energy that accounts for the real spectral distribution of the vibrational density of state (VDOS) in the electron-phonon coupling. The results in Fig. 3 point out a particularly low $h \nu$ value with respect to those previously reported $(37 \mathrm{meV}$ instead of $79 \mathrm{meV}$ ). This result suggests that the low-energy VDOS excess, typical of amorphous structures, ${ }^{24,25}$ is enhanced with respect to pure silica, because of either the networking peculiarities of the sol-gel synthesis or fluorine modifications of the medium range structure. Indeed, networking and fluorine effects on the soft phonon modes were respectively observed in sol-gel silica ${ }^{26}$ and F-doped CVD 
silica. ${ }^{11}$ By contrast, the $X h \nu / 2 \sigma_{0}$ contribution from static disorder is $29 \mathrm{meV}$, larger than $20 \mathrm{meV}$ of pure CVD silica, indicating that fluorine introduction through sol-gel synthesis does not give rise to a decreased structural disorder. Therefore, the small contribution from dynamical disorder is the main cause of the Urbach energy and high-energy shift of the absorption edge in this material. Lower $h \nu$ and larger $X h \nu / 2 \sigma_{0}$ values indicate that the fluorine modified sol-gel synthesis gives rise to a less rigid glassy structure without ordering effects. This is consistent with the low structural stress encountered in silica from sol-gel synthesis and responsible for the higher radiation hardness of pure sol-gel silica. $^{14}$

The authors acknowledge DESY and the European Community support for measurements at HASYLAB under Contract No. RII3-CT-2004-506008 (IA-SFS), and financial support under EU under Contract No. MRTN-CT-2006036040.

${ }^{1}$ M. Rothschild, T. M. Bloomstein, J. E. Curtin, D. K. Downs, T. H. Fedynyshyn, D. E. Hardy, R. R. Kunz, V. Liberman, J. H. C. Sedlacek, R. S. Uttaro, A. K. Bates, and C. Van Peski, J. Vac. Sci. Technol. B 17, 3262 (1999).

${ }^{2}$ V. Liberman, T. M. Bloomstein, M. Rothschild, J. H. C. Sedlacek, R. S. Uttaro, A. K. Bates, C. Van Peski, and K. Orvek, J. Vac. Sci. Technol. B 17, 3273 (1999).

${ }^{3}$ M. Rothschild, T. M. Bloomstein, T. H. Fedynyshyn, V. Liberman, W. Mowers, R. Sinta, M. Switkes, A. Grenville, and K. Ortek, J. Fluorine Chem. 122, 3 (2003).

${ }^{4}$ C. M. Smith and L. A. Moore, J. Fluorine Chem. 122, 81 (2003).

${ }^{5}$ K. Saito and A. Ikushima, J. Appl. Phys. 91, 4886 (2002).

${ }^{6}$ K. Ishii, A. Takami, and Y. Ohki, J. Appl. Phys. 81, 1470 (1997).
${ }^{7}$ M. Mizuguchi, L. Skuja, H. Hosono, and T. Ogawa, J. Vac. Sci. Technol. B 17, 3280 (1999).

${ }^{8}$ L. Skuja, K. Kajihara, Y. Ikuta, M. Hirano, and H. Hosono, J. Non-Cryst. Solids 345-346, 328 (2004).

${ }^{9}$ S. Hayakawa and L. L. Hench, J. Non-Cryst. Solids 262, 264 (2000).

${ }^{10}$ R. E. Youngman and S. Sen, J. Non-Cryst. Solids 349, 10 (2004).

${ }^{11}$ N. Shimodaira, K. Saito, N. Hiramitsu, S. Matsushita, and A. J. Ikushima, Phys. Rev. B 71, 024209 (2005).

${ }^{12}$ C. J. Brinker and G. W. Scherer, Sol-Gel Science (Academic, New York, 1989), p. 97.

${ }^{13}$ S. Agnello, N. Chiodini, A. Paleari, and A. Parlato, J. Non-Cryst. Solids 353, 573 (2007).

${ }^{14}$ E. Vella, R. Boscaino, G. Navarra, and B. Boizot, J. Non-Cryst. Solids 353, 559 (2007).

${ }^{15}$ E. N. Poddenezhny, Mater. Sci. 353, 573 (2007).

${ }^{16}$ Y. Morimoto, S. Nozawa, and H. Hosono, Phys. Rev. B 59, 4066 (1999).

${ }^{17}$ K. Tsukuma, N. Yamada, S. Kondo, K. Honda, and H. Segawa, J. NonCryst. Solids 127, 191 (1991).

${ }^{18}$ F. L. Galeener, Phys. Rev. B 19, 4292 (1979).

${ }^{19}$ E. M. Rabinovich, D. L. Wood, D. W. Johnson, Jr., D. A. Vincent, and J. B. Macchesney, J. Non-Cryst. Solids 82, 42 (1986).

${ }^{20}$ K. M. Davis, A. Agarwal, M. Tomozawa, and K. Hirao, J. Non-Cryst. Solids 203, 27 (1996).

${ }^{21}$ L. Skuja, Defects in $\mathrm{SiO}_{2}$, Related Dielectrics: Science, Technology, edited by G. Pacchioni, L. Skuja, and D. L. Griscom (Kluwer, Dordrecht, 2000), Vol. II/2, p. 73 .

${ }^{22}$ K. Saito and A. Ikushima, Phys. Rev. B 62, 8584 (2000).

${ }^{23}$ G. D. Cody, T. Tiedje, B. Abeles, B. Brooks, and Y. Goldstein, Phys. Rev. Lett. 47, 1480 (1981)

${ }^{24}$ R. O. Pohl, X. Liu, and E. J. Thompson, Rev. Mod. Phys. 74, 991 (2002).

${ }^{25}$ O. Pilla, S. Caponi, A. Fontana, J. R. Gonçalves, M. Montagna, F. Rossi, G. Viliani, L. Angelani, G. Ruocco, G. Monaco, and F. Sette, J. Phys.: Condens. Matter 16, 8519 (2004).

${ }^{26}$ A. Bortolotta, G. Carini, G. D'Angelo, M. Ferrari, A. Fontana, M. Montagna, F. Rossi, and G. Tripodo, J. Non-Cryst. Solids 280, 249 (2001). 